\title{
Early detection of fetal micropenis after IVF-ICSI
}

\author{
Asher Bashiri, Reli Hershkovitz \\ Moshe Mazor, Jordana Mashiach Friedler
}

\begin{abstract}
Introduction: The term micropenis is applied if the measured penile length is more than 2.5 standard deviations below the mean for the age. Early detection of fetal micropenis is important for the etiology and early treatment. Case report: We present a case of a primiparous woman after IVF-ICSI (in vitro fertilization-intracytoplasmatic sperm injection), in whom on early anatomy scan fetal micropenis was suspected. The patient had an amniocentesis and the fetal karyotype was $46 \mathrm{XY}$. The patient decided to continue the pregnancy and was followed-up closely. Severe isolated micropenis was confirmed after a normal vaginal delivery. The baby received Human Chorionic Gonadotropin (HCG) for three months without improvement and underwent three operations with partial improvement. Conclusion: This case emphasizes the importance of early anatomy scan and its ability to diagnose malformations including those of the genital system especially after IVF-ICSI.
\end{abstract}

Asher, Bashiri ${ }^{1}$, Reli Hershkovitz ${ }^{1}$, Moshe Mazor $^{1}$ Jordana Mashiach Friedler ${ }^{1}$

Affiliations: ${ }^{1}$ Department of Obstetrics and Gynecology, Soroka University Medical Center, Faculty of Health Sciences, Ben-Gurion University of the Negev, BeerSheva, Israel.

Corresponding Author: Asher Bashiri, Department of Obstetrics and Gynecology, Soroka University Medical Center, P.O Box 151, Beer-Sheva64101, Israel; Ph: +972-8-640-5151; Fax: +972-8-640-3294 Email: abashiri@bgu.ac.il

Received: 07 April 2011

Accepted: 16 May 2011

Published: 01 October 2011
Keywords: Ultrasound, Fetal micropenis, Early anatomy scan, IVF-ICSI

$* * * * * * * * *$

Bashiri A, Hershkovitz R, Mazor M, Friedler JM. Early detection of fetal micropenis after IVF-ICSI. International Journal of Case Reports and Images 2011;2(10):1-5.

$* * * * * * * * *$

doi:10.5348/ijcri-2011-10-57-CR-1

\section{INTRODUCTION}

The term micropenis (MP) is applied if the measured penile length is more than 2.5 standard deviations below the mean for age. Micropenis may be isolated or part of a syndrome. Most cases are due to lack or decreased testosterone levels that can be secondary to lack of gonadotropins or testosterone deficiency or dysfunction. The treatment of micropenis should include a course of HCG or testosterone after delivery and it may play an important role in the outcome. Early diagnosis and evaluation can help parents and clinicians in decision making and management. The purpose of this case report is to describe the ultrasonographic early detection of micropenis in a fetus after IVF-ICSI.

\section{CASE REPORT}

A 27-year-old healthy primiparous woman, after IVF-ICSI, had normal nuchal translucency of the fetus, at 13 weeks gestational age. At 15.5 weeks, in the early anatomy scan, a male fetus was diagnosed and the penis length was measured $1.5 \mathrm{~mm}$. A micropenis was 
suspected. No other malformations were found. The patient was referred to a tertiary medical center and a multidisciplinary consultation was done including genetic consultation. The patient refused detailed evaluation except for an amniocentesis and a $46 \mathrm{XY}$ karyotype was found. At 20.5 weeks gestational age, a late anatomy scan was done and the penis length was $2.4 \mathrm{~mm}$. At 23 weeks it was $3.5 \mathrm{~mm}$, at 28 weeks 4.2 $\mathrm{mm}$ and at 32 weeks $5.5 \mathrm{~mm}$, all of which were $<3^{\text {rd }}$ percentile (Figure 1, 2, 3). At 38 weeks gestational age, the patient delivered vaginally, a 2956 gram male neonate with Apgar scores of 6 and 9 at 5 and 10 minutes, respectively. In the neonatal department, physical examination revealed a penis length of approximately $8 \mathrm{~mm}$ with bilateral cryptorchidism (Figure 4). The testosterone concentration was 3 $\mathrm{ng} / \mathrm{ml}$. Human chorionic gonadotropin (HCG) treatment was started and circumcision was delayed. Several operations were performed with partial successes. Now this patient is lost to follow up. This case is unique in the very early diagnosis of severe micropenis.

\section{DISCUSSION}

Fetal gender assignment is attempted during prenatal ultrasound evaluation not only due to parent's curiosity but also for identification of normal gender development $[1,2]$.

It is the autonomy based right of pregnant woman to be routinely offered information about fetal sex identification in an ultrasound examination although it may raise ethical challenges especially in specific social circumstances and countries in which sex ratio imbalances bring forth to serious social consequences [3].

Prenatal determination of fetal gender by ultra sound during the second and third trimesters of pregnancy is based on the demonstration and measurement of the size of the penis in the male or labial folds in the female. However, there is no appreciable difference in the size of the penis and the clitoris until after 14 weeks of gestation [4]. In 1989, Emerson and colleagues described the "sagittal sign" as a sonographic marker for the prediction of fetal gender starting from the $14^{\text {th }}$ week of gestation [5]. An alternative to this method by which fetal gender can be assigned at 11-14 weeks of gestation was offered by Effrat et al. in 1999 [6]. This is done by examining the genital region in a mid sagittal plane with the fetus horizontal (parallel) to the probe in a supine position with no extension of the limbs or spine, a photograph is taken and the angle of the genital tubercle to horizontal line through the lumbosacral skin surface is measured. The fetal gender is assigned male if the angle is greater than $30^{\circ}$ and female if the phallus was parallel or convergent (less than $30^{\circ}$ ) to the horizontal line. Using this method, gender assignment has a high accuracy rate at 12-14 weeks, $99.6 \%$ for males and $97.4 \%$ for females. The accuracy of female assignment increased with

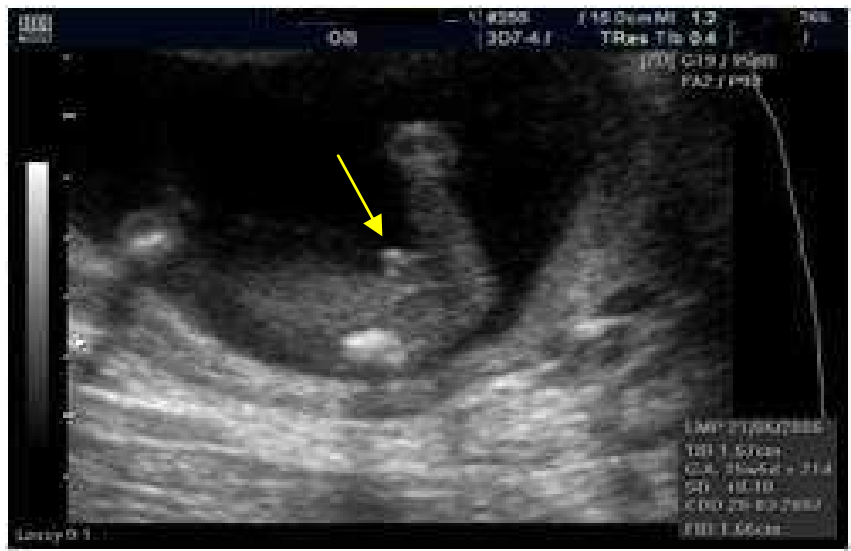

Figure 1: Fetal micropenis at 15.5 weeks gestation.

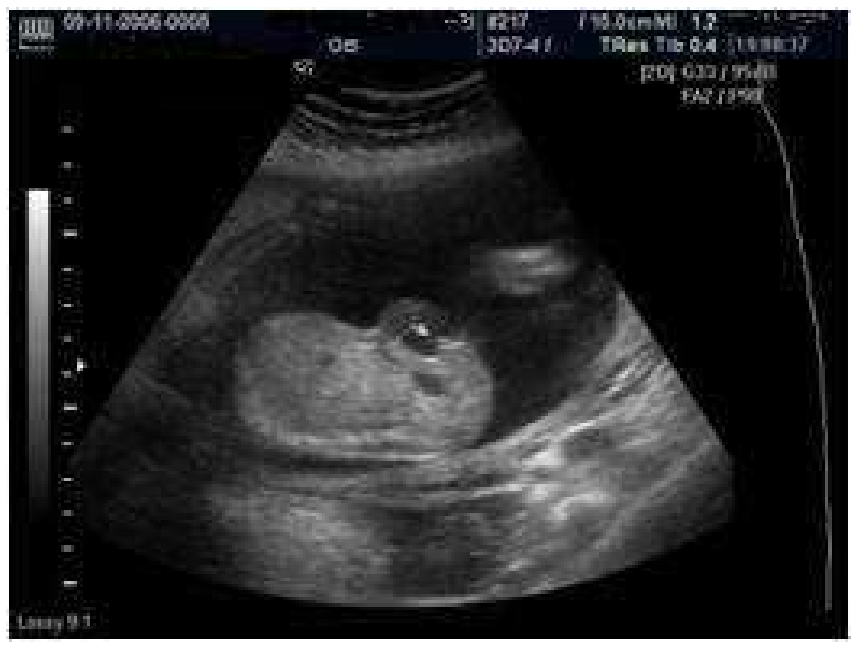

Figure 2: Fetal micropenis at 22 weeks gestation.

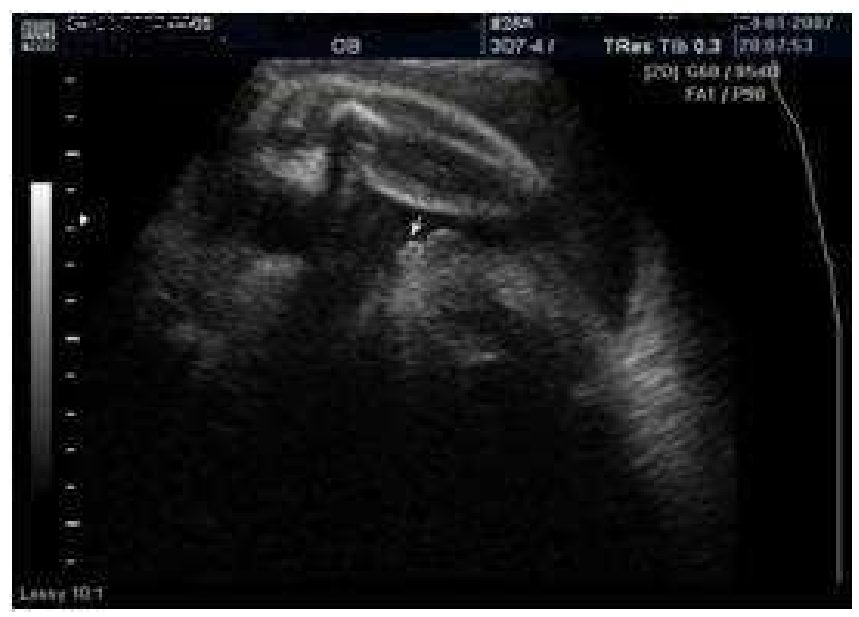

Figure 3: Fetal micropenis at 32 weeks gestation.

increasing CRL (crown rump length). The accuracy of male assignment did not change significantly with 


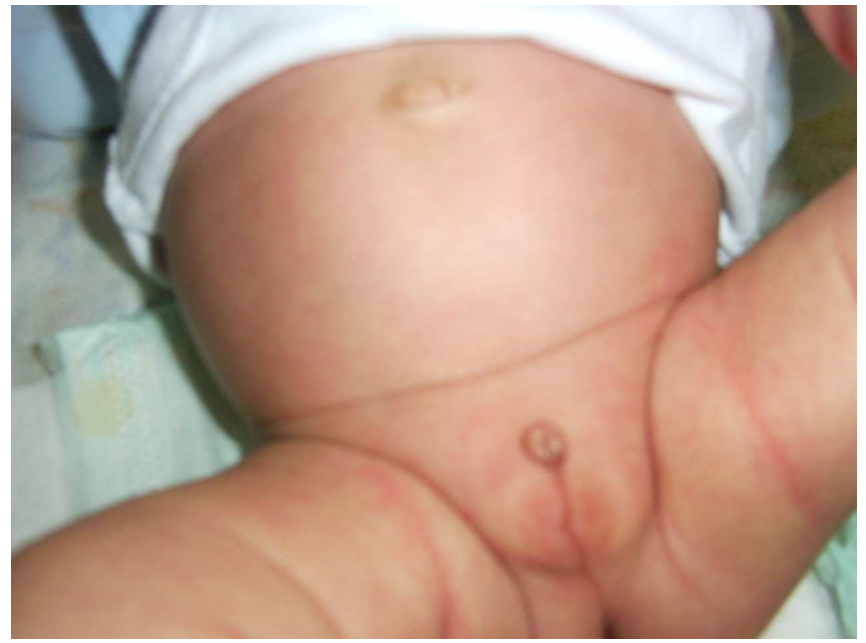

Figure 4: Micropenis at birth

an increase in CRL [7].

Several authors $[8,9]$ published normograms for penile length during normal gestation. Penile size correlates to gestational age and there is no difference in penile length if measured before or after bladder emptying. Abnormal values may assist in prenatal diagnosis of mciropenis and the possibility of associated syndromes. Zalel et al. offer a regression equation for penile size as function of gestational age (GA): (square root) penile length $(\mathrm{mm})=0.277+0.121$ $x$ GA (week). The correlation coefficient $r=0.967$, was found to be highly statistically significant $(\mathrm{P}<0.0001)$ [8].

In humans, sexual development is genetically determined and requires appropriate differentiation of the gonads in order to acquire a sexually dimorphic phenotype. Secretion of at least three testicular hormones that include: Anti-Mullerian hormone $(\mathrm{AMH})$, Testosterone ( $\mathrm{T}$ ), and Insulin Like Hormone (INSL 3) is necessary for proper male sexual development [10].

In full term neonates, micropenis is defined as 2.5 $\mathrm{SD}$ below the mean penile length for age. This means a penile length of less than $2-2.5 \mathrm{~cm}$. There are more than 70 genetic/chromosomal abnormalities in which ambiguous genitalia and or micropenis/ hypogenitalism are a frequent or occasional part of the syndrome [11].

Differential diagnosis for MP is heterogeneous. It can appear isolated or as part of a syndrome. If the MP is an isolated finding, the etiology is usually endocrinological - and is a result of low levels of testosterone that can be secondary to lack of gonadotrophins. Other causes include decrease in testosterone production or peripheral production of dehydroxytyestosterone, low sensitivity to testosterone and lack of growth hormone (GH).

Conditions that are associated with hypogonadotrophic hypogonadism are: lack of several hypophyseal hormones secondary to a genetic mutation. Conditions in which there is a decrease in testosterone production include: Anorchia which is the atrophy of the testicles after the $12^{\text {th }}$ week. Conditions where there is a decrease in peripheral testosterone production includes partial 17 $\beta$-hydroxysteroid dehydrogenase and $5 \alpha$-reductase deficiency.

There are several genetic syndromes that are associated with MP such as Kallman syndrome, Kliefenter syndrome, Bardet-Biedl syndrome, Noonnan syndrome, CHARGE syndrome, Robinow syndrome, Prader-Willi syndrome and Septo-optic dysplasia [12 - 15].

The work-up when MP is suspected following ultrasonography (US) should be multidisciplinary and include a geneticist, endocrinologist, and urologists as well as obstetric ultrasound follow up. The tests that are included in the evaluation are:

1. Karyotype for detection of genetic sex - this will differentiate between male MP to a female with abnormal genitalia. Karyotype will also help in diagnosis of associated genetic syndromes such as Prader-Willi.

2. Gonadotropin levels including $\mathrm{GH}$, cortisol, glucose and thyroide function.

3. Testosterone and Dehydro-testosterone before or after HCG injection.

4. Imaging: US to look for uterus and ovaries in female virilization cases and MRI in hypopituitarism.

5. Other tests: Dynamic test to determine the ability and the production of FSH and LH by the hypophysis.

Some of these tests can be performed during pregnancy and several closely after delivery. Another recent aspect that has been investigated is the association between genital malformations and IVFICSI pregnancies as in our case. It is known that endogenous testosterone contributes to postnatal phallic growth [16]. Mau Kai and colleagues reported low testosterone levels in infant boys conceived by Intracytoplasmic Sperm Injection (ICSI) and found significantly but only slightly smaller penile length in ART (artificial reproductive technology) boys done due to reasons other than male factor. The significance of this is yet unknown. They found a significant reduction (27\%) in serum testosterone levels and a significantly higher LH to testosterone ratio in boys conceived by ICSI compared with normal control boys [17]. These point towards a subtle impairment of Leydig cell function in boys after IVF-ICSI using sperm from oligozoospermic men. A large register based study demonstrated a significantly higher prevalence of hypospadias among ICSI boys compared with normal control boys [18]. This is in line with the relatively high prevalence of hypospadias (1.6\%) in ICSI boys compared with normal control boys (1.2\%), although this difference was not statistically significant [18]. 
Initial treatment of MP is a short course of testosterone to assess the ability of the penis to respond to the hormone. Testosterone can be given via intramuscular injections or applied topically. Testosterone cypionate or enanthate in oil, $25 \mathrm{mg}$, is given intramuscularly every three weeks for four months for the initial course. The adverse effects are minimal and include temporary accelerated growth velocity and bone age [19]. Topical testosterone application also has been shown to be effective in young children. Arisaka et al. administered 5\% testosterone ointment daily to 50 boys aged five months to eight years for 30 days, which resulted in increased penile length. Transdermally absorbed testosterone also has been shown to stimulate the secretion of growth hormone from the pituitary gland, which increases the production of insulin like growth factor-I, a factor that promotes bone growth. Longterm administration has the potential to enhance penile length as well as skeletal growth [20]. If the micropenis does not achieve adequate length through medical intervention one may turn to surgical options after all other treatments have been exhausted. The surgical options are highly complex and carry with them many risks, especially because the patient most likely will undergo multiple treatments and the results may not produce acceptable functional or cosmetic outcomes [21]. Social and psychological concerns justify early palliative phalloplasty. Circumcision should be postponed when male sex is diagnosed and the child will be brought up as a male.

The prognosis for MP secondary to GP deficiency or testosterone is good. They usually respond to testosterone treatment and behave normally in adulthood. Follow up for this child is necessary because of abnormal growth and developmental abnormalities.

\section{CONCLUSION}

The demonstration of the genital area during ultrasound anatomy scan is essential and important to determine normal anatomy and development. Early detection of micropenis requires detailed evaluation and multidisciplinary approach. Special notice should be given in ART conceived fetuses.

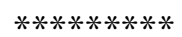

\section{Author Contributions}

Asher Bashiri - Substantial contributions to conception and design, Acquisition of data, Drafting the article, Revising it critically for important intellectual content, Final approval of the version to be published

Reli Hershkovitz - Substantial contributions to conception and design, Drafting the article, Final approval of the version to be published
Moshe Mazor - Substantial contributions to conception and design, Drafting the article, Final approval of the version to be published

Jordana Mashiach Friedler - Substantial contributions to conception and design, acquisition of data, Drafting the article, revising it critically for important intellectual content, Final approval of the version to be published

\section{Guarantor}

The corresponding author is the guarantor of submission.

\section{Conflict of Interest}

Authors declare no conflict of interest.

\section{Copyright}

(C) Asher Bashiri et al. 2011; This article is distributed under the terms of Creative Commons attribution 3.0 License which permits unrestricted use, distribution and reproduction in any means provided the original authors and original publisher are properly credited. (Please see www.ijcasereportsandimages.com /copyright-policy.php for more information.)

\section{REFERENCES}

1. Achiron R, Pinhas-Hamiel O, Zalel Y, Rostein Z, Lipitz S. Development of fetal male gender: Prenatal sonographic mesurment of the scrotum and evaluation of testicular descent. Ultrasound Obstet Gynecol. 1998;11:242-5.

2. Soriano D, Lipitz S, Seidman DS, Maimon R, Mashiach S, Achiron R. Development of the fetal uterus between 19 and 38 weeks gestation in-utero ultrasonic measurements. Humen Reprod. 1999;14:215-18.

3. Chervenk FA, McCullough LB. Sex determination by ultrasound: ethical challenges of sex ratio imbalances and invidious discrimination. Ultrasound Obstet Gynecol. 2009;34:245-246.

4. Feldman KW, Smith DW. Fetal phallic growth and penile standards for newborn male infants. J Pediatr. 1975;86:395-8.

5. Emerson DS, Falker RE, Brown DL. The Sagittal sign. An early second trimester sonographic indicator of fetal gender. $J$ Ultrasound Med. 1989;8:293-7.

6. Effrat Z, Akinfenwa O, Nicolaides H. First-trimester determination of fetal gender by ultrasound. Ultrasound Obstet Gynecol. 1999;13:305-7.

7. Effrat Z, Perri T, Ramati E, Tugendrich D, Meizner I. Fetal Gender assignment by first-trimester ultrasound. Ultrasound Obstet Gynecol. 2006;27: 619-21.

8. Zalel Y, Pinhas-Haimel O, Lipitz S, Mashiach S, Achiron R. the development of the fetal penis - an in utero sonographic evaluation. Ultrasound Obstet Gynecol. 2001;17:129-31.

9. Johnson P, Maxwell D. Fetal penile length. Ultrasound Obstet Gynecol. 2000;15:308-10. 
10. Nef S, Prada LF Hormones in male sexual development. Genes Dev. 2000;14:3075-86.

11. Jones K. Ambiguous genitalia and micropenis. In: Smith DW, eds. Reconizable patterns of human malformations. Philadelphia: W.B. Saunders, 1997:835-6.

12. Achermann JC, Ozisik G, Meeks JJ, Jameson JL. Genetic causes of human reproductive disease. J Clin Endocrinol Metab. 2002;87:2447-54.

13. Sykiotis GP, Hoang XH, Avbelj M, Hayes FJ, Thambundit A, Dwyer A, Au M, Plummer L, Crowley WF Jr, Pitteloud N. Congenital Idiopathic Hypogonadotropic Hypogonadism: Evidence of Defects in the hypothalamus, pituitary, and testes.. J Clin Endocrinol Metab. 2010;95:3019-27.

14. Gad YZ, Nasr H, Mazen I, Salah N, el-Ridi R5 alphareductase deficiency in patients with micropenis..J Inherit Metab Dis. 1997;20:95-101.

15. Ragen DC, Cassie AJ, Rink RC Genitourinary anomalies in the CHARGE association. J Urol. 1989;181:622-5.

16. Chellakooty M, Skakkebaek NE, Toppari J, Main, KM. Postnatal penile length and growth rate correlate to serum testosterone levels: a longitudinal study of 1962 normal boys. Eur J Endocrinol. 2006;154:125-129.

17. Claudia Mau Kai, Katharina M. Main, Anders Nyboe Andersen, Anne Loft, Niels E. Skakkebæk, Anders Juul. Reduced Serum Testosterone Levels in Infant Boys Conceived by Intracytoplasmic Sperm Injection J Clin Endocrinol Metab. 2007;92:25982603.

18. Kallen B, Finnstrom O, Nygren KG, Olausson PO In vitro fertilization (IVF) in Sweden: risk for congenital malformations after different IVF methods. Birth Defects Res A Clin Mol Teratol. 2005;73:162-169.

19. Menon, P, Khatwa, U. The child with micropenis. Indian Journal of Pediatrics 2000;67:455-460.

20. Arisaka O, Hoshi M, Kanazawa S, Nakajima D, Numata M, Kuribayashi T, et al. Systemic effects of transdermal testosterone for the treatment of microphallus in children. Pediatrics International 2001;43;134-6.

21. Shirley Tsang. When Size Matters: A Clinical Review of Pathological Micropenis. J Pediatr Health Care. 2010;24:231-40. Epub 2009 Jul 23. 